\title{
Effect of microwave field on the emergence of the electromotive force in asymmetrical $p-n$-junction
}

\author{
Muhammadjon Gulomkodirovich Dadamirzaev \\ Namangan Engineering Pedagogical Institute, 716,003 Namangan, Uzbekistan
}

\section{Email address:}

dadamirzaev70@mail.ru

\section{To cite this article:}

Muhammadjon Gulomkodirovich Dadamirzaev. Effect of Microwave Field on the Emergence of the Electromotive Force in Asymmetrical P-N-Junction. American Journal of Electromagnetics and Applications. Vol. 2, No. 5, 2014, pp. 45-48. doi: 10.11648/j.ajea.20140205.12

\begin{abstract}
In asymmetric p-n-junction in a strong microwave field for the analysis of voltage and current necessary to consider both the heating of electrons and holes. total current and voltage generated is determined not by the temperature of the hot electrons and the temperature of the carriers that are decisive. three-dimensional image on the surface $f\left(j_{s c}, T_{e} T_{b}\right)$ and $f\left(U_{o c}\right.$ $T_{e} T_{h}$ ) to determine the possible range of the voltages and currents generated by the p-n-junction in a strong microwave field.
\end{abstract}

Keywords: P-N-Junction, Microwave Field, Emf, CVC P-N-Junction

\section{Introduction}

The nature of the external forces that give rise to EMF in semiconductors can be established by examining them in elementary quantum processes. Lasing and recombination processes in semiconductors are the main quantum processes that determine the occurrence of electromotive forces. The study of these processes in semiconductors with potential barriers in strong electromagnetic fields allows us to construct a correct theory of transport phenomena and may contribute to the development of new semiconductor devices.

According to the concept of elementary excitations of a semiconductor is the electron-phonon and hole gas. Electromagnetic wave excites an electron-hole and phonon system. In pulse mode, the microwave exposure primarily heats the electron-hole system.

Mechanisms of EMF and currents in the p-n-junction in a strong microwave (MW) fields in the pulsed mode are studied in [1]. Study of p-n-junctions in microwave fields shows that the generated current and voltage in the silicon p-n-junction is much more than a germanium junction. The authors [2] believe that the values of the observed large abnormal current and emf depends on the recombination processes in the space charge region. On the basis of theoretical studies [3] it is shown that the open circuit voltage $U_{o c}$ asymmetrical p-n junction $\left(\mathrm{p}_{\mathrm{p}}>>\mathrm{n}_{\mathrm{n}}\right)$, located in a microwave field, determined by the modulation potential barrier height and temperature of holes. However, in these studies scouted EMF and current three-dimensional image of the surface $f\left(j_{\text {кз }}, T_{e}, T_{h}\right)$ и $f\left(U_{x x}\right.$, $\mathrm{T}_{\mathrm{e}}, \mathrm{T}_{\mathrm{h}}$, in the microwave field.
In papers $[2,4]$ investigated asymmetrical $\mathrm{p}$-n-junction in a strong microwave field, when the concentration of holes in $p$ region is much greater than the concentration of electrons in the n-region $p_{p}>>n_{n}$, nn here and $\mathrm{pp}$ - the concentration of majority carriers p- and n- regions. However, in [2] does not take into account the change in temperature of the holes, considering these changes is small compared with the changes in the electron temperature. On the basis of theoretical studies [5], the open circuit voltage $U_{o c}$ asymmetric p-n- transition $\left(p_{p} \gg n_{n}\right)$, located in a microwave field, determined by the modulation potential barrier height and temperature of the hole, despite the fact that $T_{e}>T_{h}$. In [6] studied the currents and the emf arising from asymmetrical p-n-junction under the influence of a strong microwave field. In [4, 7] in the study of the temperature dependence of the diode ideality CVC $m=f\left(T_{e}\right)$ believe that $T_{h}=$ const, and the study of function $m=f\left(T_{h}\right)$ was considered $T e=$ const. In the study of the temperature dependence of the open circuit voltage of the temperature of the electrons and holes were made the same assumption. In a real experiment, they never met. In fact, while changing the temperature of the electrons and holes temperature. Therefore, the analysis of the experimental results should be considered as simultaneous heating of electrons and holes.

The relevance of these tasks and interest in them due to the extensive use of modern technology in diodes and transistors, under the influence of strong electromagnetic fields, the socalled microwave diodes and microwave transistors. These 
tasks will be needed in the development of new types of devices that require operation of the microwave diodes as generators and detectors.

The aim of this work is to study the influence of the microwave field on the electromotive force in asymmetric $\mathrm{p}$ $\mathrm{n}$-junction in the three-dimensional coordinates

\section{Effect of Microwave on the Occurrence of Voltage in Asymmetric P-N-Junction}

The average value of the total current through the diode when exposed to electromagnetic wave consists of the electron and hole currents [5].

At low power UHF waves can be neglected heating of electrons and holes. Then the current through the diode occurs only due to rectification. [7]

At high power wave when $T_{e} \neq T_{h} \neq T$, CVC is determined by the formula [5]:

$$
\begin{aligned}
& \bar{j}=j_{s e}\left\{\left(\frac{T_{e}}{T}\right)^{\frac{1}{2}} \exp \left(\frac{e \varphi_{0}}{k T}-\frac{e\left(\varphi_{0}-U\right)}{k T_{e}}\right)_{0}^{2 \pi} \exp \left(-\frac{e U_{B} \cos (\omega t)}{k T_{e}}\right) \frac{d(\omega t)}{2 \pi}-1\right\}+ \\
& +j_{s h}\left\{\left(\frac{T_{h}}{T}\right)^{\frac{1}{2}} \exp \left(\frac{e \varphi_{0}}{k T}-\frac{e\left(\varphi_{0}-U\right)}{k T_{h}}\right)_{0}^{2 \pi} \exp \left(-\frac{e U_{B} \cos (\omega t)}{k T_{h}}\right) \frac{d(\omega t)}{2 \pi}-1\right\},
\end{aligned}
$$

where, $j_{s e}=\frac{e D_{e} n_{p}}{L_{e}}, j_{s h}=\frac{e D_{h} p_{n}}{L_{h}}$ - saturation currents for the electron and hole currents, $\varphi 0$ - height of the potential barrier in the absence of an electromagnetic wave; U-voltage appearing across the diode, $U_{B}=-\int_{0}^{d} E_{\mathrm{B}} d x-\mathrm{AC}$ voltage of the incident wave, created by the barrier diode, $\mathrm{T}$ is the temperature of the lattice, k-Boltzmann constant, Te and Th temperature of electrons and holes, Eb-wave electric field, e is the charge of an electron, De and Dh - diffusion coefficients of electrons and holes, Le and Lh - the diffusion length of electrons and holes, $\mathrm{np}$ and $\mathrm{pn}$ - the concentration of minority carriers. From (1) it is evident that the diode current is increased by modulating the potential barrier height.

Consider the short-circuit current $(U=0)$. From the formula (1) that the short-circuit current is equal to

$$
\begin{aligned}
& \bar{j}=j_{s e}\left\{\left(\frac{T_{e}}{T}\right)^{\frac{1}{2}} \exp \left[\frac{e \phi_{0}}{k}\left(\frac{1}{T}-\frac{1}{T_{e}}\right)\right] \int_{0}^{2 \pi} \exp \left(-\frac{e U_{B} \cos (\omega t)}{k T_{e}}\right) \frac{d(\omega t)}{2 \pi}-1\right\}+ \\
& +j_{s h}\left\{\left(\frac{T_{h}}{T}\right)^{\frac{1}{2}} \exp \left[\frac{e \phi_{0}}{k}\left(\frac{1}{T}-\frac{1}{T_{h}}\right)\right]_{0}^{2 \pi} \exp \left(-\frac{e U_{B} \cos (\omega t)}{k T_{h}}\right) \frac{d(\omega t)}{2 \pi}-1\right\}
\end{aligned}
$$

This shows that the short-circuit current is also growing due to modulation of the potential barrier height. Modulation of the potential barrier height always increases the short circuit current of the $\mathrm{p}$-n-junction in a microwave field.

Consider now the open-circuit voltage $U_{o c}(\mathrm{j}=0)$. Using formula (1) we find:

$$
\begin{aligned}
& \frac{e D_{e} n_{p}}{L_{e}}\left\{\left(\frac{T_{e}}{T}\right)^{\frac{1}{2}} \exp \left(\frac{e \varphi_{0}}{k T}-\frac{e\left(\varphi_{0}-U_{O C}\right)}{k T_{e}}\right) \int_{0}^{2 \pi} \exp \left(-\frac{e U_{B} \cos (\omega t)}{k T_{e}}\right) \frac{d(\omega t)}{2 \pi}-1\right\}= \\
& =-\frac{e D_{h} p_{n}}{L_{h}}\left\{\left(\frac{T_{h}}{T}\right)^{\frac{1}{2}} \exp \left(\frac{e \varphi_{0}}{k T}-\frac{e\left(\varphi_{0}-U_{O C}\right)}{k T_{h}}\right)_{0}^{2 \pi} \exp \left(-\frac{e U_{B} \cos (\omega t)}{k T_{h}}\right) \frac{d(\omega t)}{2 \pi}-1\right\}
\end{aligned}
$$

As can be seen from the expression (3) open-circuit voltage $U_{o c}$ determined by the concentration, temperature, diffusion coefficients and the diffusion length of minority carriers. It follows that it is necessary to take into account all the factors responsible for the change in current through the p-n-junction when exposed to strong electromagnetic free.

If we consider that for the concentration of minority carriers:

$$
\mathrm{p}_{\mathrm{n}}=\frac{\mathrm{n}_{\mathrm{i}}^{2}}{\mathrm{n}_{\mathrm{n}}} ; \mathrm{n}_{\mathrm{p}}=\frac{\mathrm{n}_{\mathrm{i}}^{2}}{\mathrm{p}_{\mathrm{p}}}
$$

here ni - intrinsic concentration of charge carriers;

The diffusion coefficients for electrons and holes:

$$
D_{e}=\frac{k T_{e}}{e} \mu_{e}, \quad D_{h}=\frac{k T_{h}}{e} \mu_{h}
$$

Diffusion length for electrons and holes:

$$
L_{e}=\sqrt{D_{e} \tau_{e}}, \quad L_{h}=\sqrt{D_{h} \tau_{h}}
$$

For the dependence of the mobility of electrons and holes from the lattice temperature [8]:

$$
\begin{array}{ll}
\mu_{\mathrm{e}}=4 \cdot 10^{9} \cdot \mathrm{T}^{-2,6} & \sim 300<\mathrm{T}<400 \mathrm{~K} \\
\mu_{\mathrm{h}}=2,5 \cdot 10^{8} \cdot \mathrm{T}^{-2,3} & \sim 150<\mathrm{T}<400 \mathrm{~K}
\end{array}
$$

Depending private carrier concentration in the $\mathrm{Si}$ lattice temperature [8]:

$$
\begin{aligned}
& \mathrm{n}_{\mathrm{i}}^{2}=1,5 \cdot 10^{33} \cdot \mathrm{T}^{3} \cdot \mathrm{e}^{-\frac{\Delta \varepsilon}{\mathrm{kT}}} \\
& \text { где } \quad \Delta \varepsilon=1,27 \ni \mathrm{B}
\end{aligned}
$$

Then, for the saturation current of electron and hole currents using (4) - (8) formula finds the following:

$$
\begin{aligned}
& \mathrm{j}_{\mathrm{se}}=\frac{\mathrm{eD} \mathrm{D}_{\mathrm{p}}}{\mathrm{L}_{\mathrm{e}}}=\mathrm{e} \frac{\mathrm{D}_{\mathrm{e}}}{\mathrm{L}_{\mathrm{e}}} \frac{\mathrm{n}_{\mathrm{i}}^{2}}{\mathrm{p}_{\mathrm{p}}}=\mathrm{e} \frac{\mathrm{D}_{\mathrm{e}}}{\sqrt{\mathrm{D}_{\mathrm{e}} \tau_{\mathrm{e}}}} \frac{\mathrm{n}_{\mathrm{i}}^{2}}{\mathrm{p}_{\mathrm{p}}}=\mathrm{e} \sqrt{\frac{\mathrm{D}_{\mathrm{e}}}{\tau_{\mathrm{e}}}} \cdot \frac{\mathrm{n}_{\mathrm{i}}^{2}}{\mathrm{p}_{\mathrm{p}}} \\
& =\mathrm{e} \sqrt{\frac{\frac{\mathrm{kT}}{\mathrm{e}} \mu_{\mathrm{e}}}{\tau_{\mathrm{e}}} \cdot \frac{1,5 \cdot 10^{33} \cdot \mathrm{T}^{3} \cdot \mathrm{e}^{-\frac{\Delta \varepsilon}{\mathrm{kT}}}}{\mathrm{p}_{\mathrm{p}}}}= \\
& =\sqrt{\frac{\mathrm{e}^{2} \mathrm{kT}_{\mathrm{e}}}{\mathrm{e} \tau_{\mathrm{e}}} 4 \cdot 10^{9} \cdot \mathrm{T}^{-2,6}} \cdot \frac{1,5 \cdot 10^{33} \cdot \mathrm{T}^{3} \cdot \mathrm{e}^{-\frac{\Delta \varepsilon}{\mathrm{kT}}}}{\mathrm{p}_{\mathrm{p}}}= \\
& \sqrt{\frac{\mathrm{ekT} T_{\mathrm{e}}}{\tau_{\mathrm{e}}} \cdot \mathrm{T}^{-2,6} \mathrm{~T}^{6} \cdot 10^{9}} \cdot \frac{2 \cdot 1,5 \cdot 10^{33} \cdot \mathrm{e}}{\mathrm{p}_{\mathrm{p}}}= \\
& =\sqrt{\frac{\mathrm{ekT} \mathrm{e}_{\mathrm{e}}}{\tau_{\mathrm{e}}} \cdot \mathrm{T}^{3,4} \cdot 10^{9}} \cdot \frac{3 \cdot 10^{33} \cdot \mathrm{e}^{-\frac{\Delta \varepsilon}{\mathrm{kT}}}}{\mathrm{p}_{\mathrm{p}}}
\end{aligned}
$$




$$
\begin{aligned}
& \mathrm{j}_{\mathrm{se}}=\sqrt{\frac{\mathrm{ekT}}{\tau_{\mathrm{e}}} \cdot \mathrm{T}^{3,4} \cdot 10^{9}} \cdot \frac{3 \cdot 10^{33} \cdot \mathrm{e}^{-\frac{\Delta \varepsilon}{\mathrm{kT}}}}{\mathrm{p}_{\mathrm{p}}} \\
& j_{\text {sh }}=\frac{e D_{h} p_{n}}{L_{h}}=e \frac{D_{h}}{L_{h}} \frac{n_{i}^{2}}{n_{n}}=e \frac{D_{h}}{\sqrt{D_{h} \tau_{h}}} \frac{n_{i}^{2}}{n_{n}}=e \sqrt{\frac{D_{h}}{\tau_{h}}} \cdot \frac{n_{i}^{2}}{n_{n}} \\
& =\mathrm{e} \sqrt{\frac{\frac{\mathrm{kT}}{\mathrm{e}} \mu_{\mathrm{h}}}{\tau_{\mathrm{h}}}} \cdot \frac{1,5 \cdot 10^{33} \cdot \mathrm{T}^{3} \cdot \mathrm{e}^{-\frac{\Delta \varepsilon}{\mathrm{kT}}}}{\mathrm{n}_{\mathrm{n}}}= \\
& =\sqrt{\frac{\mathrm{ekT}}{\tau_{\mathrm{h}}} 2,5 \cdot 10^{8} \cdot \mathrm{T}^{-2,3}} \cdot \frac{1,5 \cdot 10^{33} \cdot \mathrm{T}^{3} \cdot \mathrm{e}^{-\frac{\Delta \varepsilon}{\mathrm{kT}}}}{\mathrm{n}_{\mathrm{n}}} \\
& =\sqrt{\frac{\mathrm{ekT}_{\mathrm{h}}}{\tau_{\mathrm{h}}} \cdot \mathrm{T}^{-2,3} \mathrm{~T}^{6} \cdot 2,5 \cdot 10^{9}} \cdot \frac{1,5 \cdot 10^{33} \cdot \mathrm{e}^{-\frac{\Delta \varepsilon}{\mathrm{kT}}}}{\mathrm{n}_{\mathrm{n}}}= \\
& =\sqrt{\frac{\mathrm{ekT}_{\mathrm{h}}}{\tau_{\mathrm{h}}} \cdot \mathrm{T}^{3,7} \cdot 2,5 \cdot 10^{8}} \cdot \frac{1,5 \cdot 10^{33} \cdot \mathrm{e}^{-\frac{\Delta \varepsilon}{\mathrm{kT}}}}{\mathrm{n}_{\mathrm{n}}} ; \\
& \mathrm{j}_{\mathrm{sh}}=\sqrt{\frac{\mathrm{ekT}_{\mathrm{h}}}{\tau_{\mathrm{h}}} \cdot \mathrm{T}^{3,7} \cdot 2,5 \cdot 10^{8}} \cdot \frac{1,5 \cdot 10^{33} \cdot \mathrm{e}^{-\frac{\Delta \varepsilon}{\mathrm{kT}}}}{\mathrm{n}_{\mathrm{n}}}
\end{aligned}
$$

Using (9) and (10) using Formula (2) define a threedimensional plot of the short circuit current of the electron temperature and the holes (Fig. 1).

Fig. 1 that the fault current is strongly dependent on the temperature of the holes and is almost independent of the electron temperature and it lies on the surface of the above $f\left(j_{\kappa 3}, T_{e}, T_{h},\right)$.

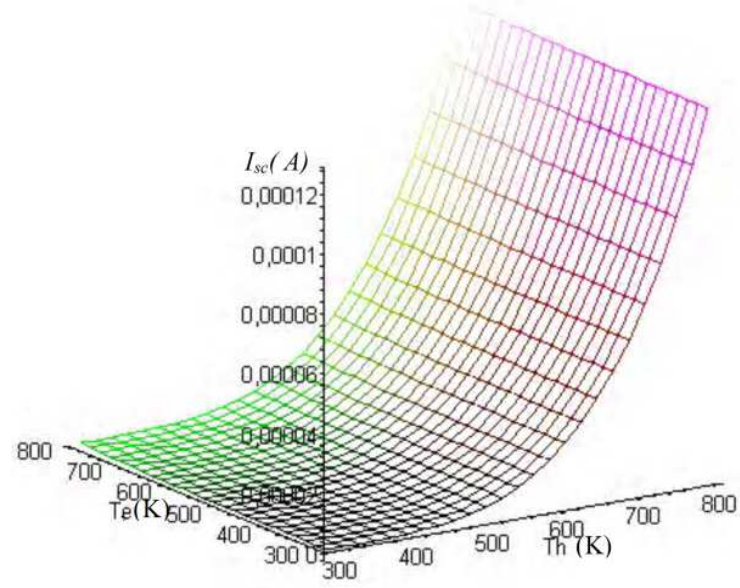

Fig. 1. Dependence of short circuit current on the temperature of the electrons and holes

Formula (3) allows analyzing graphically the dependence of $\mathrm{f}\left(U_{o c}, T_{e}, T_{h}\right)$. Fig. 2 shows a three-dimensional image of the surface $\mathrm{f}\left(U_{o c}, T_{e}, T_{h}\right)$, shows the voltage value at the surface which may be $U_{o c}$, depending on the temperatures Te and Th. Thus, in asymmetric p-n-junction in a strong microwave field for the analysis of voltage and currents must be considered as the heating of electrons and holes.

Using formula (1), taking into account the formula (4-8) investigated the effect of heating the charge carriers in the CVC p-n-junction in a strong microwave field. First, assuming a constant temperature of holes analyzed CVC p-njunction temperature change of the electrons. Thus, it follows that the increase in the current of hot carriers in asymmetric p-n-junction $\left(p_{p}>>n_{n}\right)$ is almost independent of the electron temperature.

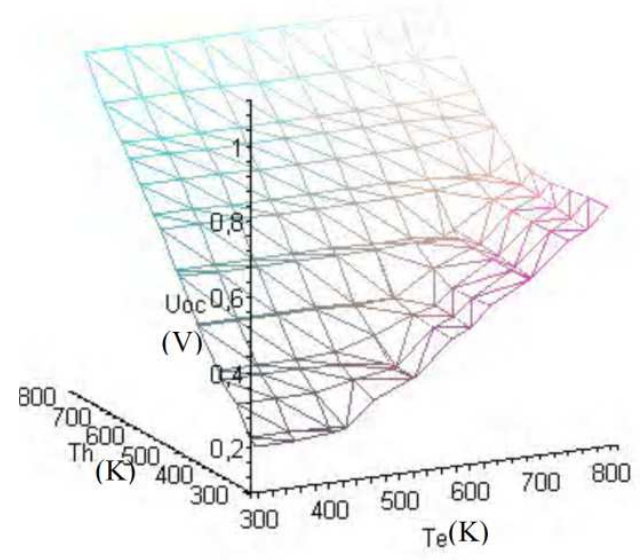

Fig. 2. Dependences of the open circuit voltage $U_{o c}$ temperature of electrons and holes.

Figure 3 shows the I-V characteristics of p-n-junction in three-dimensional space $\left(j, U, T_{h}\right)$ at a constant temperature of electrons $(T e=$ const $)$.

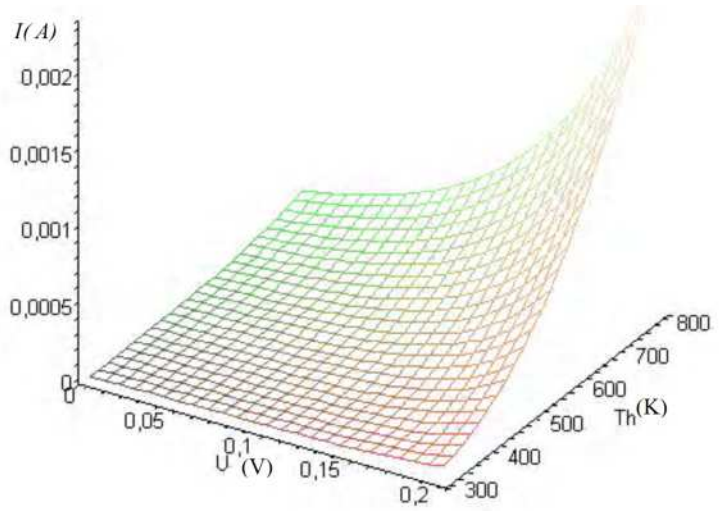

Fig. 3. I-V characteristics of an asymmetric p-n-junction in the threedimensional coordinates $(j, U, T h)$ at a constant temperature of electrons (Te = const).

As can be seen from the graph, the temperature increases with holes to $800 \mathrm{~K} 300 \mathrm{~K}$ current increases more than an order of magnitude. Such a strong dependence of the current temperature of holes indicates that the main current is determined by the hole current. The line of intersection of Fig.3 and Fig.4 should give real CVC p-n-junction for different microwave power wave. From Figure 3 it is seen that when $T_{h}=$ const and $n_{n} \ll p_{p}$ CVC diode remains almost unchanged despite the fact that the electron temperature increases nearly three-fold. Such insensitivity to temperature $\mathrm{CVC}$ electrons due to the fact that the current through the p-n-junction is determined by a hole current $\left(j_{n}\right.$ $\ll j_{p}$ ), as the temperature of holes at the same interval affects approximately CVC procedure. 


\section{Comparison of the Theoretical Results with Experiments}

We obtained the dependence of the open circuit voltage of Uoc electron temperature $T e$ at constant temperature $T_{h}$ holes [5], as well as the open-circuit voltage dependence on temperature $U_{o c}$ holes at a constant electron temperature $T_{e}$ [5]. In the experimental work [2] shows the dependence of the electron temperature $T_{e}$ and the open circuit voltage of the microwave power $U_{o c}$ wave. Using these experimental data the dependence of the open circuit voltage $U_{o c}$ of the electron temperature $T_{e}$. The dependence of the open circuit voltage $U_{o c}(\mathrm{~B})$ on the electron temperature $T_{e}(\mathrm{~K})$ coincides qualitatively with the theoretical results obtained by us [5]. For currents in the $\mathrm{p}$-n-junction placed in a strong microwave field, the temperature of the holes is decisive.

\section{Conclusion}

The mechanism of the occurrence of unbalanced currents in silicon p-n-junction, analyze their temperature dependence. It is shown that for the calculation of the CVC p-n-junction is necessary to consider not only the increase in the electron temperature, and the temperature of the hole. It is established that the CVC diode determined by the temperature of the carrier that carries the main current through the p-n-junction. Analyze CVC p-n-junction in three $\left(j, U, T_{e}\right)$ coordinate at a constant temperature of holes $\left(T_{h}=\right.$ const $)$ and at a constant temperature of electrons $\left(T_{e}=\right.$ const $)$. Three-dimensional $\left(j_{s c}\right.$, $T_{e} T_{h}$ ) plot of short circuit current on the temperature of the electrons and holes, and also shows the three-dimensional image of the surface $\left(U_{o c}, T_{e}, T_{h}\right.$ ). The theoretical results are compared with experiment.Литература:

\section{References}

[1] G.Gulyamov, M.G.Dadamirzaev, S.R.Boydedaev. Emf of hot carriers due to the modulation of the surface potential in a strong microwave field. // FTP - St. Petersburg, 2000. - T.34. №5. - P. 572-575.

[2] Ablyazimova NA, Veynger AI, nutrition V.Elektricheskie properties of silicon p-n-junctions in strong microwave fields // Semiconductors. - St. Petersburg, 1988.- T.22. Number 11 S.2001-2007.

[3] M.G.Dadamirzaev. Warming up of the charge carriers and rectification current asymmetric p-n-junction in the microwave electromagnetic field-fi. // FTP - St. Petersburg, 2011. - T.45. №3. - P. 299-302.

[4] Shamirzaev SH, Guliamov G. Dadamirzaev MG, Guliamov AG Ideality factor of the current-voltage characteristics of $p$ $\mathrm{n}$-junctions in the strong field of the microwave // Physics and Technology poluprovodnikov- St. Petersburg, 2009.- T.43, №1. - P. 53-57.

[5] MG Dadamirzaev Warming up of the charge carriers and rectification current asymmetric p-n-junction in the microwave electromagnetic field // Semiconductors. - St. Petersburg, 2011. - T.45. №3. - P. 299-302.

[6] MG Dadamirzaev Heating of electrons and holes in asymmetric p-n-junction, located in a microwave field // Physical Engineering surface. -Ukraine, 2013. -t.11, №2, s.191-193.

[7] Shamirzaev SH, Guliamov G. Dadamirzaev MG, Guliamov AG Electromotive force at rectifying barrier in microwave electromagnetic field // Physical Zhurnal. Uzbek Tashkent, 2009. -11 (№2) .- S.122-127.

[8] P.I.Baransky, V.P.Klochkov, I.V.Potykevich. Semiconductor electronics. Handbook. (Kyiv., Naukova Dumka, 1975) s.704. 